\title{
Klet Observatory - European Contribution to Detecting and Tracking of Near Earth Objects
}

\author{
Milos Tichy \\ Klet Observatory, Zatkovo nabrezi 4, CZ-370 01 Ceske Budejovice, South Bohemia, Czech Republic \\ Email: mtichy@klet.cz
}

Keywords: astrometry, Near Earth Objects, telescope

\begin{abstract}
Near Earth Object (NEO) research is an expanding field of astronomy. Is is important for solar system science and also for protecting human society from asteroid and comet hazard. A near-Earth object (NEO) can be defined as an asteroid or comet that has a possibility of making an approach to the Earth, or possibly even collide with it. The discovery rate of current $N E O$ surveys reflects progressive improvement in a number of technical areas. An integral part of NEO discovery is astrometric follow-up fundamental for precise orbit computation and for the reasonable judging of future close encounters with the Earth including possible impact solutions. A wide international cooperation is fundamental for NEO research.
\end{abstract}

The Klet Observatory (South Bohemia, Czech Republic) is aimed especially at the confirmation, early follow-up, long-arc follow-up and recovery of Near Earth Objects. It ranks among the world's most prolific professional NEO follow-up programmes.

The first NEO follow-up programme started at Klet in 1993 using 0.57-reflector equipped with a small CCD camera. A fundamental upgrade was made in 2002 when the 1.06-m KLENOT telescope was put into regular operation. The KLENOT Telescope is the largest telescope in Europe used exclusively for observations of minor planets (asteroids) and comets and full observing time is dedicated to the KLENOT team.

Equipment, technology, software, observing strategy and results of both the Klet Observatory NEO Project between 1993-2010 and the first phase of the KLENOT Project from March 2002 to September 2008 are presented. They consist of thousands of precise astrometric measurements of Near Earth Objects and also three newly discovered Near Earth Asteroids.

Klet Observatory NEO activities as well as our future plans fully reflect international strategies and cooperation in the field of NEO studies.

\section{Introduction}

NEO research is an expanding field of astronomy, important both for solar system science and for protecting human society from the asteroid and comet hazard. NEOs are sources of impact risk and represent usually a low-probability but potentially a very high-consequence natural hazard. Studies of NEOs contributed significantly to our overall understanding of the Solar System, its origin and evolution. On the Earth surface there is possible to find impact craters. Impact craters are geologic structures formed when an asteroid or comet colide with the Earth. All bodies in the solar system have been heavily bombarded by meteoroids, or 
asteroids, throughout their history. The surfaces of the Moon, Mars and Mercury, where other geologic processes stopped hundreds millions of years ago, record this bombardment clearly. On the Earth, however, which has been even more heavily impacted than the Moon, craters are continually erased by erosion and redeposition as well as by volcanic resurfacing and tectonic activity. Thus only about 120 terrestrial impact craters have been recognized, the majority in geologically stable cratons of North America, Europe and Australia where most exploration has taken place. We know also impacts on planets in the Solar system in recent years (Jupiter on July 1994, impact with comet Shoemaker-Levy 9), and moreover impact on the Earth (asteroid 2008 TC3, diameter of 3 meters, collide with the Earth on October 7, 2008). Therefore is necessary to study parent bodies of possible impact risk [10]. Consequently, interest in detecting, tracking, cataloguing and the physical characterizing of these bodies has continued to grow. The discovery rate of current NEO surveys refects progressive improvement in a number of technical areas.

Near Earth Objects are asteroids and comets with perihelion distance q less than $1.3 \mathrm{AU}$. The vast majority of NEOs are asteroids, referred to as Near-Earth Asteroids (NEAs). NEAs are divided into four groups (Amors, Apollos, Atens and IEOs) according to their perihelion distance, aphelion distance and their semi-major axes. There are currently more than 6000 known NEAs.

Potentially Hazardous Asteroids (PHAs) are NEAs whose Minimum Orbit Intersection Distance (MOID) with the Earth is $0.05 \mathrm{AU}$ or less and whose absolute magnitude $\mathrm{H}$ is $\mathrm{H}=$ 22.0 mag. or brighter i. e., whose estimated diameter exceeds about 140 meters. There are currently more than 1000 known PHAs.

Virtual Impactors (VIs) are asteroids for which possible impact solutions, compatible with the existing observations are known, and a very small, but definitely non-zero, probability of collision exists. Virtual Impactors are listed on the Sentry Impact Risk page hosted by the NASA/JPL [1] and the NEODys Risk Page maintained by the University of Pisa which operates the impact risk monitoring system CLOMON2 [5]. As new positional observations become available, the most likely outcome is that the object's orbit is improved, uncertainties are reduced, impact solutions are ruled out for the next 100 years and the object will eventually be removed from the lists.

A wide international cooperation and on-line sharing of permanently updated data in the framework of NEO observations is fundamental. The vast majority of permanently-updated astrometric data and orbits of asteroids and comets including Near Earth Objects goes trough the Minor Planet Center of the International Astronomical Union (Harvard Center for Astrophysics, Cambridge, MA, USA) [11], as the international clearinghouse responsible for the efficient collection, computation, checking and dissemination of these data. The first step of understanding NEO population is discovery, both Near Earth Asteroids and Near Earth Comets. The vast majority of NEO discoveries have been made by wide field telescopic surveys. The most prolific surveys are Catalina Sky Survey, LINEAR, LONEOS, NEAT and Spacewatch, all of them supported by NASA. An integral part of NEO discovery is follow-up astrometry that is required so the orbits of newly discovered objects become secure. Extensive follow-up on timescales of weeks and months is usually required for subsequent return recoveries, and may become critical in ensuring that PHAs and VIs are not lost. Calculation of precise orbits and determination of impact probabilities require enough precise astrometric measurements covering appropriate orbit arcs. Astrometric follow-up is essential also for tar- 
gets of future radar observations, space mission targets and other observing campaigns. For brighter objects many amateur volunteer observers use small or moderate-size telescopes all over the world so as to help accomplish this task. For fainter objects just several professional telescopes of 1-m class or larger are used regularly for astrometric follow-up, mainly the JPL Table Mountain 0.6-m, Mt. John 0.64-m, Klet Observatory KLENOT 1.06-m, Spacewatch 1.8-m, and Mt. Lemmon 1.5-m [2]. In recent years this work is done also with the Magdalena Ridge Observatory 2.4-m and the Astronomical Research Institute near Charleston 0.81-m telescopes. Considering a geographical distribution, the majority of them is siuated in the U.S. A good number of European follow-up observations are provided by the community of amateur observers (Great Shefford is most active of them). In addition to the Klet Observatory, just Calar Alto 1.23-m telescope serves for NEO follow-up in Europe on occasions. All measurements of astrometric coordinates on the sky (Right Ascension and Declination) are calculated as topocentric although the calculations of orbits are made geocentric. As regards close approaches to the Earth and reliable orbits computation there is necessary to have precise Earth coordinates (longitude, latitude and elevation above see level in meters).

\section{KLENOT Project}

The Klet Observatory NEO follow-up programme was started in 1993 using 0.57-m reflector equipped with SBIG CCD cameras. It was the second world most prolific NEO astrometric programme from 1998 to 2001. Considering the urgent need for astrometric follow-up of fainter and fast-moving objects as well as our experience we decided to use resources of the Klet Observatory (South Bohemia, Czech Republic) for building a 1-meter size telescope for such purposes. This KLENOT telescope was put into operation in 2002 [6]. We report the results obtained during six years of regular KLENOT operation as well as future plans based on technical improvement of the KLENOT system and also inspired by the planned next generation surveys.

\section{KLENOT Project goals}

The KLENOT project is a project of the KLEt observatory Near earth and Other unusual objects observations Team (and Telescope). Our observing strategy is to concentrate particularly on fainter objects, up to a limiting magnitude of $\mathrm{m}(\mathrm{V})=22.0 \mathrm{mag}$. Reasonable object selection is a key part of the observation planning process. Therefore the main goals of The KLENOT Project have been selected as:

\section{Confirmatory observations of newly discovered fainter NEO candidates}

The majority of newly discovered objects which, on the basis of their motion or orbit, appear to be NEOs as well as objects that are suspected to be comets go on promptly to the NEO Confirmation Web Page (NEOCP) maintained by the Minor Planet Center (MPC) [3]. Such NEO candidates need rapid astrometric follow-up to confirm both their real existence as a solar system body and the proximity of their orbits to that of the Earth. Some of new search facilities produce discoveries fainter than $\mathrm{m}(\mathrm{V})=20.0 \mathrm{mag}$. which need a larger telescope for confirmation and early follow-up. A 1-m class telescope is also very suitable for confirmation of very fast moving objects and our larger field of view enables to search for NEO candidates having a larger ephemeris uncertainty. 


\section{Follow-up astrometry of poorly observed NEOs}

Newly discovered NEOs need astrometric data obtained over a longer arc during the discovery opposition when they get fainter. The highest priority has been given to Virtual Impactors and PHAs. Special attention is also given to targets of future space missions or radar observations. It is necessary to find and use an optimal observing strategy to maximize orbit improvement of each asteroid along with efficient use of observing time, because reasonable object selection is a key part of the observation planning process.

\section{Recoveries of NEOs at the second opposition}

For the determination of reliable orbits it is required to observe asteroids at more than one opposition. If the observed arc in a discovery apparition is long enough, the chance for a recovery at the next apparition is good. If the observed arc at a single opposition is not sufficient and the ephemeris of selected target is uncertain, then we usually plan to search along the line of variation based on data from Minor Planet Center databases (Marsden, Williams, Spahr), Lowell Observatory databases (Bowell, Koehn), and Klet Observatory databases (Tichy, Kocer). For this purpose a larger field of view is an advantage.

\section{Analysis of cometary features}

The majority of new ground-based discoveries of comets comes from large surveys devoted, predominantly, to Near Earth Asteroids. The first step in distinguishing these newly discovered members of the population of cometary bodies consists of confirmatory astrometric observations along with detection and analysis of their cometary features [8]. Timely recognition of a new comet can help in planning future observing campaigns. The following step is to pursue the behavior of cometary bodies i.e. to obtain observation data of comet outbursts and fragmentation or splitting of cometary nuclei.

\section{Search for new asteroids}

The primary goal of the KLENOT Project is astrometric follow-up of NEOs and comets. Moreover, all of obtained CCD images are processed not just for targeted objects, but also examined visually for possible unknown moving objects. This can be achieved because the effective field of view, observing time and limiting magnitude of $\mathrm{m}(\mathrm{V})=22 \mathrm{mag}$. of the KLENOT telescope enable us to find new objects. The obtained CCD images are processed with special attention to objects showing unusual motion.

\section{KLENOT telescope}

The KLENOT telescope is located at the Klet Observatory in the Czech Republic in Central Europe. The IAU/MPC observatory code is (246) Klet Observatory-KLENOT. The geographical position of the observatory is longitude $=+14^{\circ} 17^{\prime} 17^{\prime \prime} \mathrm{E}$, latitude $=+48^{\circ} 51^{\prime} 48^{\prime \prime} \mathrm{N}$, $\mathrm{h}=1068 \mathrm{~m}$ above sea level. It is situated at a rather dark site in the middle of a protected landscape area Blansky forrest (Blansky les). 
The KLENOT telescope was built between 1996-2002 using an existing dome and infrastructure of the Klet Observatory. An original mount dating from 1960s was upgraded. A new control and computer room was built on the ground floor of the dome.

The KLENOT telescope was completed using a $1.06-\mathrm{m}$ primary mirror and a primary focus corrector. The main mirror was fabricated by Carl Zeiss Jena using Sital glass (Zerodur type) and is $\mathrm{f} / 3.0$. The primary focus corrector was designed by Sincon, Turnov, Czech Republic, and was fabricated by the Optical Facility of Charles University, Prague, Czech Republic, led by Jindrich Walter. The corrector consists of four spherical lens elements. The resulting optical configuration is $\mathrm{f} / 2.7$ folded prime focus where the CCD camera is located.

The CCD camera used for the KLENOT telescope is Photometrics Series 300. The CCD chip sensor SITe SI003B contains 1024 x 1024 pixels, pixel size 24 microns, and is back illuminated with high quantum efficiency, Q.E. $>80 \%$ in range 5500-8000 Angstroems. Imaging array size is 24.6 x 24.6 millimeters. The CCD camera includes a 16-bit digitizer with full-frame readout time of 5.4 seconds and liquid nitrogen cryogenic cooling. Cryogen hold time for our 1.1 liter dewar is over 6 hours. Dark current is virtually non-existent in this camera due to operating chip temperature of $183 \mathrm{~K}$.

The field of view of the KLENOT telescope is $33 \times 33$ arcminutes using the CCD camera mentioned above. The image scale is 1.9 arcseconds per pixel. The limiting magnitude is $\mathrm{m}(\mathrm{V})=21.5$ mag. for 120 -sec exposure time in standard weather conditions.

The KLENOT telescope has been the largest telescope in Europe used exclusively for observations of minor planets and comets up to now. All the observing time is dedicated to the KLENOT team.

\section{Klet software package}

There has been developed a special software package for KLENOT at Klet. The package combines observation planning, data-acquisition, camera control and data processing tools running on Windows and Unix platforms (recently we have been using FreeBSD-amd64). The system uses client-server architecture where appropriate and most of the software is associated with a SQL database.

The SQL database stores orbital elements and other information on minor planets updated on daily basis from text-based databases; the MPC Orbit Database (MPCORB), maintained by the Minor Planet Center, and from the Asteroid Orbital Elements Database (ASTORB), created and maintained by E. Bowell at the Lowell Observatory. The asteroids listed in the Spaceguard system The Priority List and objects listed as a Virtual Impactors by SENTRY (JPL) or by CLOMON (NEODys) are flagged in the database as well for more convenient search. In addition, the database holds orbital elements and other useful data on all solar system objects discovered at Klet (database K_KLET) and also information on comets (database COMETS) created and updated from more sources by Klet. The database also contains positions, times and observed objects on all of the processed photographic plates and CCD images.

All data are stored locally in the local network of the observatory, so the system works also in off-line mode; i.e. in cases when on-line services are not available. Beside regular updates it is possible to trigger updates for all locally stored data from external sources at any time. 
Our observers are using a web-based tool called 'ephem' for observation planning. The tool allows the user to get an ephemeris for one minor planet and/or for known minor planets in specified field in the sky at given time. The objects in the output list can be reduced to objects of given magnitude and/or type; i.e. to NEAs, PHAs, TNOs, Virtual Impactors, Klet discoveries, critical list objects, unusual or distant minor planets, Trojans, Spaceguard Priority List objects and comets. Besides the designation, position in the sky, magnitude, and other usual ephemeris data, the output list also includes information on object type, ephemeris uncertainty, date of last observation, length of orbital arc used in orbit computation.

Another tool, also used in observation planning, is Program 'KLAC' - KLENOT Atlas Coeli. This GUI program shows stars and solar system objects with a line showing their daily motion across the sky in a selected region in the sky. The size of the region usually corresponds to the FOV of the telescope used so it is also used to check the telescope position during an observation. The USNO-B1.0, USNO-A2.0 and GSC star catalogues can be used within KLAC as a source of positions, magnitude estimates and proper motion of stars.

We use $\mathrm{V}++$ for $\mathrm{CCD}$ camera control. The $\mathrm{V}++$ is a precision digital imaging system developed by Digital Optics, a standard program for Photometrics CCD cameras on the Win32 platforms. For exposure control and data-acquisition a set of scripts in VPascal (buildin programming language in $\mathrm{V}++$ ) has been written. The scripts store a sequence of several CCD frames (images) in one file in TIFF format. In the header of the sequence file information about the number of frames in the sequence, time, exposure time, equipment used and other information is included.

Programs 'Blink' and 'SumViewer' are used for blinking and manipulating RAW CCD multiimage TIFFs. Two or more selected frames from the sequence can be alternatively displayed on the monitor and visually inspected. Besides blinking, the programme also allows smoothing, inverting, shifting, co-adding and zooming of image frames.

The images taken by CCD camera are then processed through the program 'Astrometry', which has been developed for the reduction of CCD images and automatic identification of stars with USNO-B1.0, USNO-A2.0 or GSC star catalogues. The images are reduced and all objects with given conditions for signal to noise ratio are found on the image. These objects are then identified with stars from the selected star catalogue. Equatorial coordinates of objects are then determined, and at the same time stars with residuals greater than 1 arcsecond are excluded automatically or/and manually and magnitude of the objects is determined. The user then selects the desired object on the image, and the program gives appropriate output data for that object directly in the MPC format. The time of observation and other information needed for the output are derived from the data stored in the header of the image file. Information about the processed CCD image (time, filename, frame number, equatorial coordinates of the center of the frame, telescope used, exposure time, position of objects on frame, etc.) are stored in the database of processed CCD images for later use, e.g. for automated precovery program.

The residuals of the measured astrometric positions are checked before they are made available to the astronomical community. The calculation of residuals is based on osculating orbital elements of the object near the current epoch, so they are acceptable mainly for the evaluation of observations. In addition the Delta- $T$ variation of the mean anomaly is determined. Checking of both residuals and the Delta-T variation in mean anomaly helps verify object 
identification.

All the programs in the system are using The Planetary and Lunar Ephemerides DE405 provided by JPL for exact determination of planetary positions.

\section{KLENOT results}

The Klet Observatory is one of the world's most prolific professional observatories producing follow-up astrometry of NEOs [7]. The KLENOT Project results presented here were obtained during the first phase of the KLENOT Project from March 2002 to September 2008. It resulted in an important contribution to the international NEO effort. The KLENOT telescope was out of operation due to dome reconstruction between May 2005 - December 2005 .

\section{NEO follow-up}

We have measured and sent to the Minor Planet Center 52,658 positions of 5,867 objects, including 13,342 positions of 1,369 NEAs, 222 of which were Potentially Hazardous Asteroids (PHAs) and 157 of which were Virtual Impactors (VIs) in the time of observations. The majority of measured NEAs were confirmatory observations and early follow-up observations of newly discovered objects presented on the NEO Confirmation Page (NEOCP) maintained by the Minor Planet Center. These astrometric observations helped both to confirm new discoveries and to extend their observed arc. Confirmatory observations were centered on newly discovered NEO candidates fainter than magnitude $\mathrm{m}(\mathrm{V})=19.5 \mathrm{mag}$. and faster moving objects. Long-arc follow up astrometry is devoted also to NEAs fainter than magnitude $\mathrm{m}(\mathrm{V})=19.5$ mag. Special consideration is given to Virtual Impactors coming from SENTRY and CLOMON automatic monitoring systems. In many cases data obtained by the KLENOT Team alone enabled to remove predicted impact solutions. These observations were included in 561 Minor Planet Electronic Circulars. Most of the Near Earth Asteroids observed have absolute magnitude $\mathrm{H}$ between 18 and 21, but some asteroids as intrinsically faint as $\mathrm{H}=$ 30 have been observed by the 1.06-m KLENOT telescope. Recovery of NEOs at the second opposition is another important goal of the KLENOT Project. In the framework of the KLENOT Project we recovered 16 NEAs (including 2 PHAs). The absolute majority of astrometric measurements have been sent to the Minor Planet Center immediately.

\section{KLENOT discoveries}

Although searching for unknown NEAs is just a complementary goal of the KLENOT project, all obtained images are checked for possible new objects. Within the framework of this project 750 small solar system bodies have been discovered up to now. These new discoveries are processed with a special reference to objects with unusual motion. Understandably, the majority of them belong to typical main-belt minor planets, although the 3 NEAs can be considered the most important discoveries of the KLENOT Project. There are two Apollotype asteroids 2002 LK and 2006 XR4 and Aten-type asteroid 2003 UT55 [9]. 
Tichy M.: Klet Observatory - European Contribution to Detecting and Tracking of Near Earth OBJeCts

\begin{tabular}{|l|l|l|l|c|l|l|c|}
\hline Designation & Type & a [AU] & e & i [deg.] & H & Arc & Closest Earth Approach \\
\hline 2002 LK & Apollo & 1.10 & 0.15 & 25 & 24.2 & 2002 June 1-8 & 0.023 AU \\
\hline 2003 UT55 & Aten & 0.98 & 0.15 & 16 & 26.8 & 2003 Oct. 26-27 & 0.0074 AU \\
\hline 2006 XR4 & Apollo & 1.04 & 0.27 & 11 & 26.2 & 2006 Dec. 15-16 & $0.00401 \mathrm{AU}$ \\
\hline
\end{tabular}

Table 1 - KLENOT Discoveries

\section{Perspectives}

The next generation surveys, such as Pan-STARRS, LSST and the Discovery Channel Telescope will change requirements for astrometric follow-up. The role of astrometric follow-up in connection with the new generation of all-sky deeper surveys should move towards faint(er) NEOs which are in urgent need of astrometric position determinations over a longer arc. Additional follow-up observations with other telescopes would also help make linkages in their archives [4]. Another important study would be the search for and analysis of possible cometary features of newly discovered bodies to understand their true nature.

A fundamental improvement of the KLENOT telescope was started in autumn 2008. It represents installation of a new computer-controlled equatorial mount for the KLENOT telescope allowing a better sky coverage, including lower solar elongations. The new mount is designed and it is in the process of being built. The original high-quality optics of the KLENOT telescope mentioned above will be used. The technical first light and testing observations of the KLENOT Next Generation started in autumn 2010. This new mount will substantially increase telescope-time efficiency, the number of observations, their accuracy and limiting magnitude.

KLENOT hardware improvement has been followed also by software improvement. It is rather demanding to teach a computer to recognize moving bodies on astronomical images. Thus this task is left to the astronomer, but there is still room for improving limiting magnitude by co-adding of KLENOT multi-TIFF images. The program developed at the Klet Observatory has been designed to support a human eye, facilitating search for fainter objects in the solar system on our images - even for those not detectable by eye. The images are initially processed for obtaining their center coordinates, then co-added on their common centre, effectively lenghtening the exposure time of the compound image. This strategy may prove useful in a search for fainter slowly moving bodies like TNOs. Faster moving objects require a different approach. Specifying the apparent motion and position angle of such an object permits us to move the images against its predicted motion, and the object emerges from the background noise, much like faint stars on a longer exposure time image. For astrometry of the object to be possible, only a chosen area of the image can be co-added this way. Then the first position is found with respect to the reference stars of the first image and every other is computed based on the target body's movement. Furthermore, since this method allows us to compensate for the target movement, it can also be used to detect cometary features or to search for new fainter cometary fragments.

\section{Conclusion}

KLENOT Project significantly participates in follow-up of PHAs and VIs. The KLENOT Telescope is the largest telescope in Europe used exclusively for astrometric observations of asteroids and comets, so observations are focused on faint and fast moving objects. KLENOT 
observations are used to confirm discovery, determine orbit or impact solution of such objects. Improvement of KLENOT Telescope will lead to enhanced output from the programme.

\section{Acknowledgement}

The work of the Klet Observatory and the KLENOT Project is funded by the South Bohemian Region. The KLENOT Project was sponsored also by The Grant Agency of the Czech Republic Reg. No. 205/98/0266, The 2000 NEO Shoemaker Grant of The Planetary Society (U.S.A.), and The Grant Agency of the Czech Republic Reg. No. 205/02/P114.

\section{References}

1. Chamberlin A. B., Chesley S. R., Chodas P. W., Giorgini J. D., Keesey M. S., Wimberly R. N. and Yeomans D. K. 2001. Sentry: An Automated Close Approach Monitoring System for Near-Earth Objects. Bull. Amer. Astron. Soc. 33:1116

2. Larson S. 2007. Current NEO Surveys. Proceedings of the IAU Symp 236. pp.323-328.

3. Marsden B.G. and Williams G. V. 1998. The NEO confirmation page. Planetary and Space Science 46:299-302.

4. McMillan R. S. and the Spacewatch Team. 2007. Spacewatch preparations for the era of deep all-sky surveys. Proceedings of the IAU Symp 236. pp.329-340.

5. Milani A., Chesley S. R., Sansaturio A., Tommei G. and Valsecchi G. G. 2005. Nonlinear Impact monitoring: Line Of Variation searches for impactors, Icarus 173:362-384

6. Ticha J, Tichy M. and Kocer M. 2002. KLENOT - KLET OBSERVATORY NEAR EARTH AND OTHER UNUSUAL OBJECTS OBSERVATIONS TEAM AND TELESCOPE. ESA SP-500: ACM 2002. pp.793-796.

7. Ticha J., Tichy M. and Kocer M. 2007. NEO-related scientific and outreach activities at KLENOT. Proceedings of the IAU Symp 236. pp.371-376.

8. Tichy M., Ticha J. and Kocer M. 2005. Confirmation of Comet Discoveries. International Comet Quarterly 27:87-92

9. Ticha J., Tichy M., Kocer M., Honkova M. KLENOT Project 2002-2008, Meteoritics and Planetary Science, vol. 44 (2009), Issue 12, p.1889-1895

10. http://neo.jpl.nasa.gov - NASA NEO Office

11. http://minorplanetcenter.net/iau/mpc.html - Minor Planet Center 
